\title{
Téoros
}

Revue de recherche en tourisme

\section{La demande de transport}

Choix des modes de transport pour les voyageurs américains se

rendant au Québec : tendances et perspectives

\section{Jean Stafford}

Volume 6, numéro 1, mars 1987

Tourisme et transports

URI : https://id.erudit.org/iderudit/1080523ar

DOI : https://doi.org/10.7202/1080523ar

Aller au sommaire du numéro

Éditeur(s)

Université du Québec à Montréal

ISSN

0712-8657 (imprimé)

1923-2705 (numérique)

Découvrir la revue

Citer cet article

Stafford, J. (1987). La demande de transport : choix des modes de transport pour les voyageurs américains se rendant au Québec : tendances et perspectives. Téoros, 6(1), 26-42. https://doi.org/10.7202/1080523ar d'utilisation que vous pouvez consulter en ligne.

https://apropos.erudit.org/fr/usagers/politique-dutilisation/ 


\title{
Choix des modes de transport pour les voyageurs américains se rendant au Québec: tendances et perspectives
} La demande de transport

\author{
par Jean Stafford*
}

Voies d'eau, routes de terre, ports, routes de cailloux, rails, routes d'asphalte, autoroutes, corridors aćriens forment la trame des sociétés d'hier comme de celles d'aujourd'hui'1). L'histoire des sociétés nord-américaines est le récit d'une lutte sans fin pour apprivoiser l'espace, le réduire, $\mathrm{I}^{+}$animer.

L'évolution sociale et économique de la société québecoise a été étroitement dépendante des differents moyens de trans$\operatorname{potr}^{(2)}$. De la méme façon, l'industrie touristique québécoise s'est toute entière construite en fonction de ces voies de pénétration naturelles, puis artificielles, véritable ossature de ce développement du tourisme.

Le touriste est un grand consommateur d'espace; il consomme à la fois l'espace réel et l'espace ludique. L'espace récl doit devenir ludique et l'espace ludique doit devenir réel et cette inversion, toujours recommences, conditionne l'offre et la demande de transport à des fins touristiques. C'est dans ce contexte particulier qu'il faut analyser la demande de transport des visiteurs étrangers.

\section{Les États-Unis d'Amérique}

Les voyageurs qui viennent des États-Unis constituent entre $80-85 \%$, selon les périodes, des visiteurs étrangers au Québec. Ils forment donc un groupe extremement important pour l'ensemble des secteurs de l'industrie touristique. Très tôt, pour les visiteurs américains, le Québec a été une destination privilégiée; ils ont joué un grand rổle dans la création des premières grandes stations balnéaires et montagnardes ${ }^{31}$.

Entre 1920 et 1945 , les visiteurs américains ont êté à peu près les seuls "etrangers" sur nos routes et dans nos villes; la relative démocratisation de l'automobile, due à sa production massive, a elé le fer de lance de cette invasion pacifique ${ }^{[4]}$. La route devenait, et pour longtemps, le principal mode de transport pour les vacances de nos voisins du Sud.

"Economiste, professeur au Département d'etudes urbaines de I'UOAM.
Nous allons tenter ici d'analyser l'évolu= tion de la demande de transport des voyageurs en provenance des États-Unis, dans le long terme et le court terme. Il apparaît crucial de connaitue et de comprendre cette évolution; de déceler les changements sur de longues périodes et de tenter de prévoir les formes que prendra cette demande dans l'avenir proche et dans l'avenir lointain.

\section{La demande de transport}

La plupart des études sur la demande dans le secteur des transports repose implicitement sur le principe selon lequel les individus révèlent leurs préférences par les choix qu'ils effectuent face aux divers moyens de transport.

Les premiers modèles de demande étaient relativement simples; ils pouvaient se résumer à l'équation suivante:

$D_{1}=a+b_{1} C_{n}+b_{2} R_{1}+c_{t}$

où:

$\mathrm{D}=\mathrm{la}$ demande au temps $\mathrm{t}$;

$\mathrm{C}=$ le coût relatif du mode de transport au temps $\mathrm{t}$;

$\mathrm{R}=$ le niveau du revenu réel en temps $\mathrm{t}$;

$\mathrm{e}=$ un terme d'erreur;

a $=$ un terme constant.

Par la suite, des modèles plus complexes sont apparus (Wilson 1967) ( $^{(5)}$ et (Quandt, $1970)^{67}$ qui tiendront compte de plusieurs modes de transport et d'un grand nombre de relations entre des sites différents. D'autres variables telles que "la valeur du temps" "(T) et l'impact de la concurrence (") ont aussi été intégrées à ce modèle de la demande.

Enfin, d'autres chercheurs (Stophen, Meyburg, 1976) ${ }^{(10)}$ et (Rogers, Brög, 1981) (11) ont surtout étudié les principales variables reliées aux comportements mémes des voyageurs. La définition de certaines variables intéressent au plus haut point les spécialistes du tourisme; par exemple, la distinction entre voyageur et touriste, entre trajet et woyages, etc... La plupart de ces modeles quantitatifs n'ont pas tellement réussi à réduire l'ambiguilté fondamentale reliće à ces notions.
Dans l'ensemble, ces modèles exigent des données qui sont difficiles à rassembler; ils sont aussi trè complexes, longs à élaborer et à valider et posent des problèmes assez sćrieux au niveau de l'interprétation des résultats; de plus, les prévisions établies grâce à ces modeles, sont très souvent sujettes à caution. Pour toutes ces raisons, nous allons faire une étude de la demande de transport à partir de l'étude des tendances. Celles-ci peuwent être cernées de façon mathématique en comparant les diverses formes d'ajustement (linéaire, parabolique, logorithmique, logistique, etc.) ou de façon empirique (analyse de l'ćvolution d'indices et des taux d'accroissement annuel moyen, TAAM).

\section{Les tendances de longue durée}

Au-dela de la conjoncture et des cycles, il $y$ a la longue durée et celle-ci a une vie propre qui transcende l'événement quotidien. Fernand Braudel definit la tendance seculaire comme: "...une réalité que le temps use mal et véhicule très longuement"(II), pour lui "le temps court est la plus capricieuse et la plus trompeuse des durées $^{*(12)}$. Ainsi, la notion de tendance séculaire ou de tendance lourde s'inscrit dans cette longue durée; aussi "rechercher des tendances, c’est s'efforcer de décelè dans les chroniques certaines régularites, plus ou moins apparentes et simples, certains invariants liés à l'inertie des phénomènes économiques" (13).

Nous allons étudier ici les tendances lourdes de la demande de quatre modes de transport: le rail, l'automobile, l'autobus et l'avion. Dans le cas de l'automobile il s'agit de l'entrée des véhicules (et non des touristes eux-mêmes) entrant au Québec des États-Unis; pour avoir des données conparables de 1930 à 1985 , cette définition a été conservée. Dans le cas du rail, de l'autobus et de l'avion, ce sont les voyageurs étrangers entrant au Québec par les Etats-Unis pour la période 1937 à $1985^{(14)}$.

Le rail

Le plus ancien mode de transport, le rail, a connu un long déclin de 1937 à 1985 (voir graphique 1). L'année 1947 est la plus êlevé avee 244,961 voyageurs et l'année la plus faible est 1972 avec 6,546 entrées; 
l'ecart entre ces deux nombres indique bien la faiblesse d'attraction de ce mode de transport dans les dernières années. Si on considère que la moyenne des entrées pour les 49 années est de 100,812 , toutes les années, à partir de 1959 , ont ćtć bien endessous de cette moyenne de longue durée.

On peut voir, dans le tableau 1 , que les taux d'accroissements annuels moyens ${ }^{(15)}$ (moyenne géométrique) par blocs de dix années, ont été négatifs pour toutes ces périodes; la plus forte baisse, entre 1970-1979 est de $-20.6 \%$. Pour l'ensemble des périodes étudiées (1937-1985), le taux de décroissance est de $-2.7 \%$. Les chances sont très faibles pour que cette tendance séculaire se modifie dans le court et le long termes.

\section{L'automobile}

L'arrivée des véhicules des voyageurs à la frontière américaine $a$, sur 56 années, une allure un peu syncopée (voir graphique 2); on remarque une diminution importante des entrées de véhicules pendant les années 1942 à 1945. Entre les années 1945 et 1960, I'année 1957 est la valeur la plus élevée, avec 425,870 véhicules.

L'Exposition universelle de 1967 est venue brouiller les cartes; elle a gonfle artificiellement la tendance pendant les périodes 1967 à 1973; on peut considérer que la crise pétrolière a donné un coup d'arrêt à cette évolution. La moyenne des entrées de véhicules pour les 59 périodes est de 358,278 ; on peut constater que les sept dernières années (de 1979 à 1985) se situent endessous de cette moyenne globale.

On peut voir, dans le tableau no 1 , les périodes de croissance et de décroissance de cette série. Nous avons done dix années de forte croissance (1940-1949), dix années de faible décroissance (1950-1959) et encore dix années de croissance dues, en très grande partie, à la promotion de 1 'Exposition universelle; une autre période de forte décroissance de 1970-1979 et, enfin une faible croissance de 1980 à 1985 . Dans 1'ensemble, pour les 56 années, la croissance est négative avec un TAAM (moyenne géométrique) de $-0.15 \%$. La tendance de longue durée montre une lente stagnation de l'entrée des véhicules ćtrangers (1930-1985).

\section{L'autobus}

L'autobus, comme mode de transport, a connu un développement assez lent entre 1940 et 1964 (voir le graphique no 3); entre 1965 et 1974 , la progression a été extrêmement rapide. La moyenne globale pour 1940 aे 1985 est de 116,242 visiteurs; ce nombre de voyageurs n'a été atteint qu'en 1965. La crise pétrolière semble avoir renforcé la demande de ce mode de transport.

L'autobus est le seul moyen de transport dont le TAAM a été positif (voir tableau 1) durant les cinq blocs de dix années. Il

\section{GRAPHIQUE 1}

Entrées des voyagours étrangers au Québec en provenance dos Etats-Unis par le train, de 1937 a 1985.

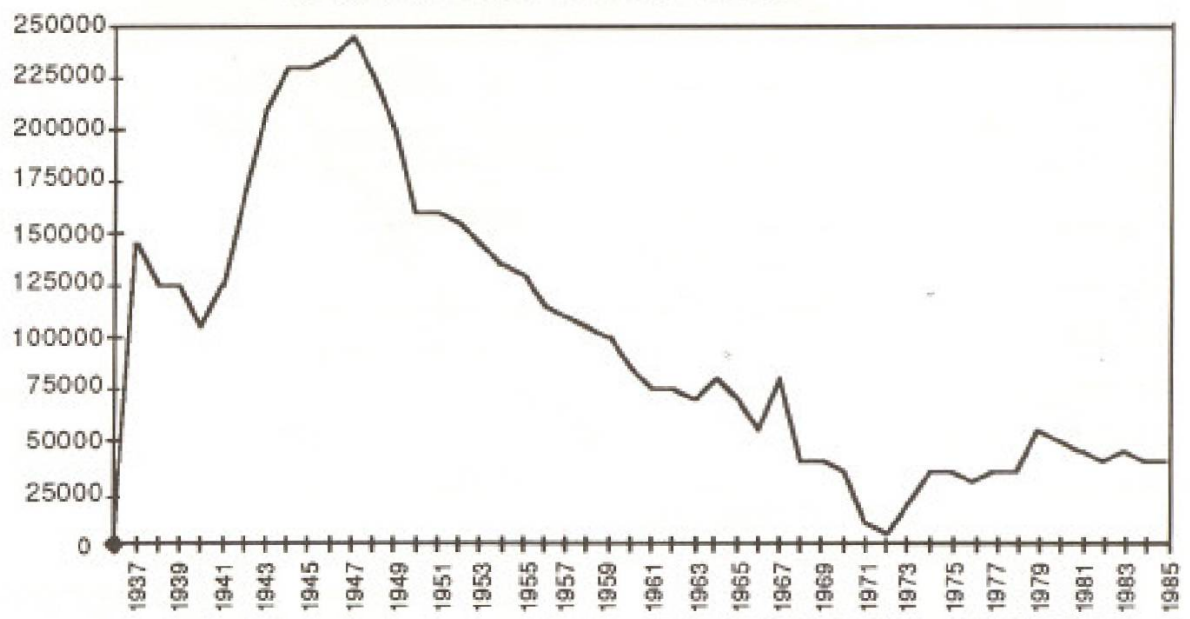

TABLEAU 1

Taux d'accroissement annuels moyens (moyenne géométrique) des entrées de voyageurs étrangers au Québec et venant des États-Unis selon divers modes de transport par blocs de dix années (1940-1985) en \%

\begin{tabular}{lrrrrr}
\hline \multicolumn{1}{c}{ Année } & $\mathbf{1 9 4 0 -}$ & $\begin{array}{c}1950- \\
1949\end{array}$ & $\begin{array}{c}1960- \\
1969\end{array}$ & $\begin{array}{l}1970- \\
1979\end{array}$ & $\begin{array}{c}1980- \\
1985\end{array}$ \\
$\begin{array}{l}\text { Mode de } \\
\text { transport }\end{array}$ & & & & & \\
\hline Rail & 6.8 & -5.6 & -8.1 & -20.6 & -4.2 \\
Véhicules (auto) & 7.2 & -1 & 7.8 & -9 & 1.8 \\
Autobus & 16.5 & 5.7 & 9.8 & 2.8 & 0.8 \\
Avion & 18.4 & 12.1 & 10.9 & -0.7 & 2.7 \\
& & & & & \\
\hline
\end{tabular}

\section{GRAPHIQUE 2}

Entrées des véhicules étrangers au Quóbec en provenance des Étals-Unis de 1930 a 1985

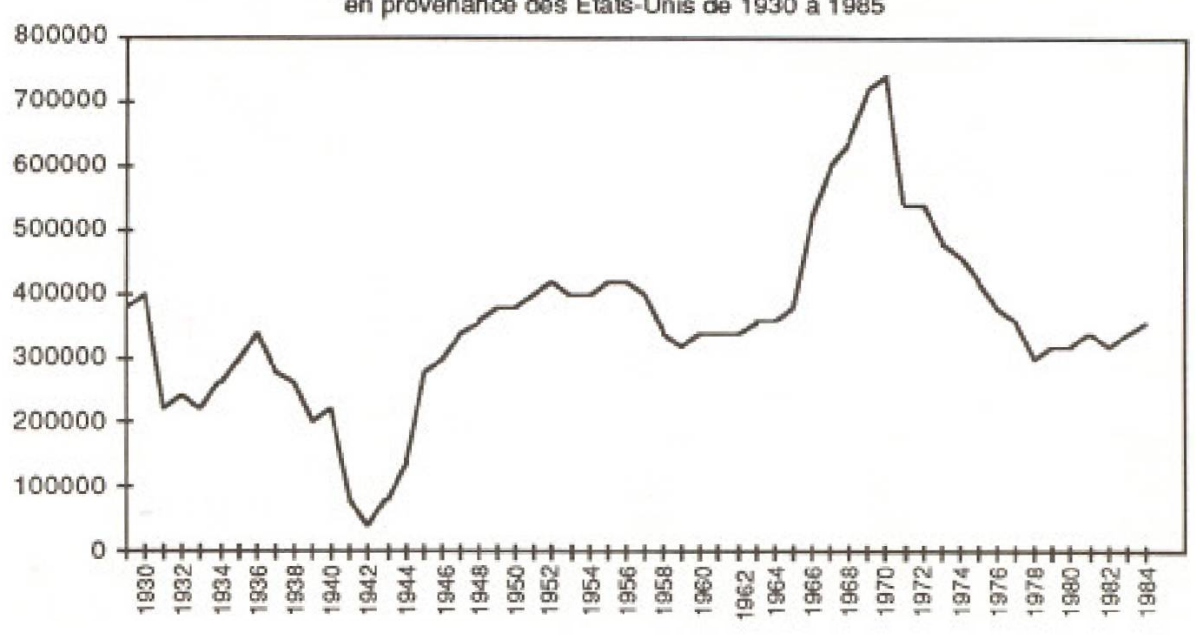


GRAPHIQUE 3

Entrées des voyageurs étrangers au Québec en provenance des Etats-Unis, par autobus, de 1940 a 1985

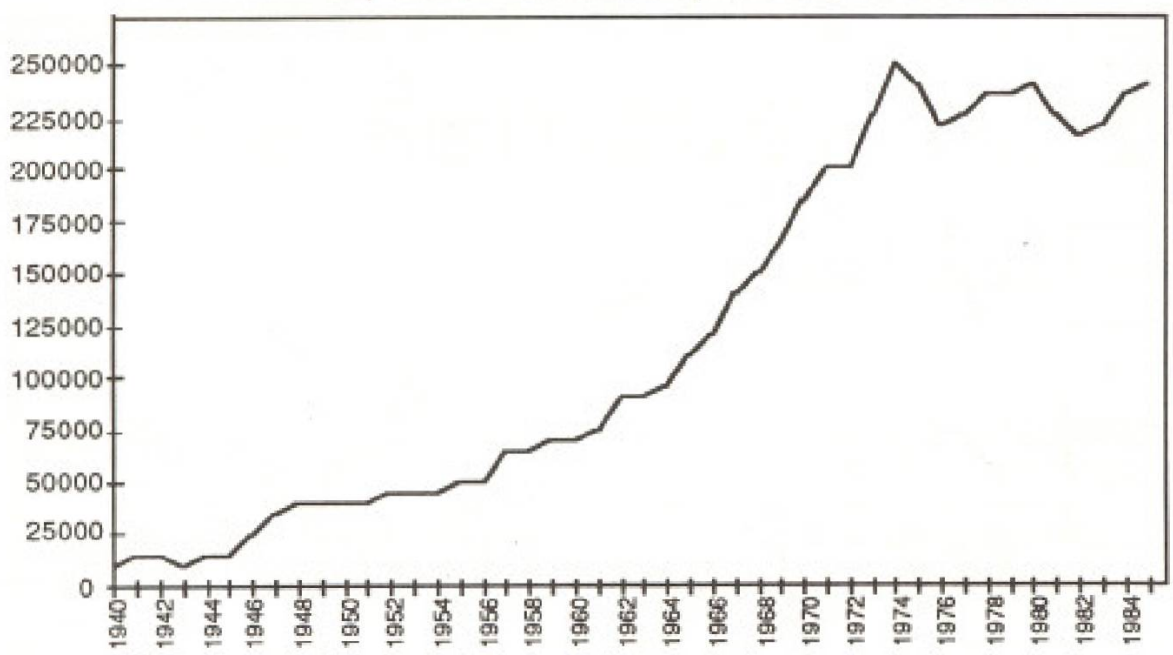

GRAPHIQUE 4

Entrées des voyageurs étrangers au Québec

en provenance des États-Unis, par avion, de 1940 à 1985

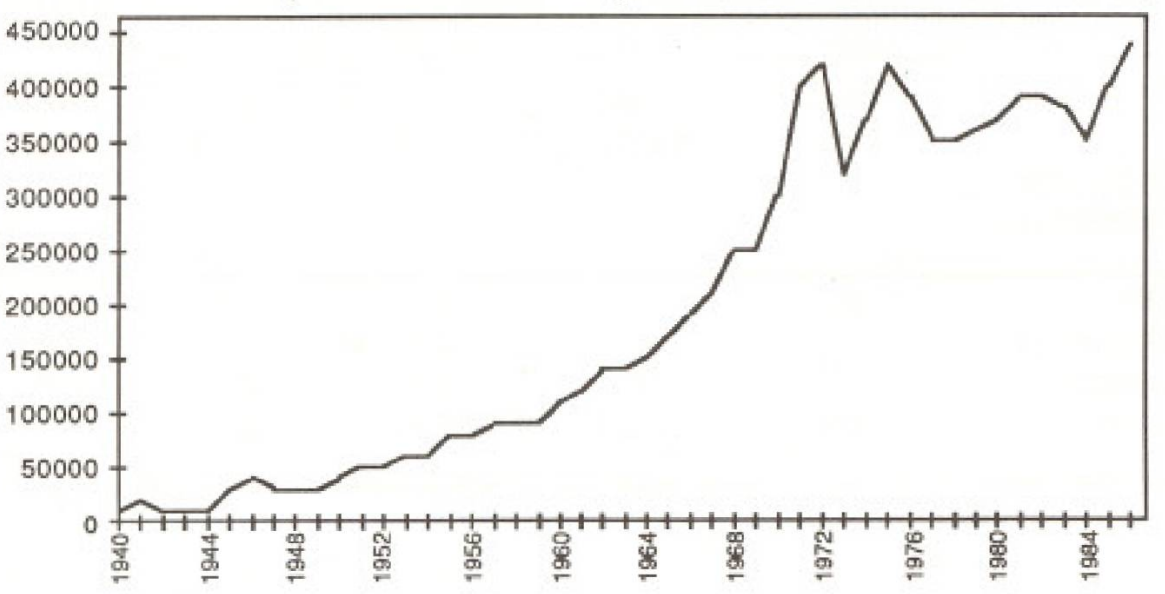

TABLEAU 2

Évolution indicielle $(1976=100)$ de la demande américaine de transport selon divers modes (auto, rail, autobus, avion)

\begin{tabular}{lrrcc}
\hline Année & Véhicules & \multicolumn{3}{c}{ Touristes } \\
\cline { 3 - 5 } & & Rall & autobus & Avion \\
\hline 1976 & 100 & 100 & 100 & 100 \\
1977 & 89 & 106 & 102 & 101 \\
1978 & 85 & 113 & 105 & 104 \\
1979 & 72 & 146 & 106 & 107 \\
1980 & 76 & 148 & 107 & 111 \\
1981 & 76 & 134 & 101 & 110 \\
1982 & 81 & 108 & 98 & 109 \\
1983 & 77 & 126 & 98 & 102 \\
1984 & 78 & 106 & 104 & 116 \\
1985 & 82 & 117 & 110 & 127 \\
\hline
\end{tabular}

faut dire que la progression de 1970 à 1979 et de 1980 à 1985 a été assez faible: $2.8 \%$ et $0.8 \%$ respectivement. On assiste là aussi à un certain ralentissement de la demande. Ce "plateau" est bien illustré dans le graphique no 3 pour les années 1974 à 1985. Pour l'ensemble des années 1940-1985, le TAAM a été de $7.6 \%$, ce qui est assez élevé.

\section{L'avion}

La demande de ce mode de transport a pratiquement doublée à toutes les cinq années, de 1940 à 1974, ce qui lui donne une forme quasi-exponentielle pour cette période (voir graphique 4). La moyenne de l'entrée des visiteurs est de 192,129 et ce palier n'a été atteint que vers 1968-1969.

On voit, dans le tableau no 1, que la croissance a été soutenue de 1940 à 1969 . Ce mode de transport est demeuré quasistationnaire entre les années 1970 et 1979 et on assiste entre 1980 et 1985 à une croissance d'environ $3 \%$ de la demande $(2.7 \%)$.

Le TAAM pour la période 1940-1985 a été de $9.5 \%$. C'est la croissance la plus forte des quatre moyens de transport pour les mêmes périodes étudiées.

\section{Comparaison des tendances de longue durée}

Les ajustements mathématiques de longue durée des différents modes de transport sont peu révélateurs. Pour le rail et l'automobile et pour toutes les formes d'ajustement utilisées (linéaire, parabolique, semilogarithmique, double-logarithmique, logistique $\left.{ }^{(16)}\right)$, les tests statistiques des équations de régression ne sont pas significatifs.

Contrairement à ce que l'on pourrait penser, ce sont les ajustements linéaires qui conviennent le mieux aux modes de transport par avion et par autobus (avec des $\mathrm{R}^{2}$ de .90 et .92 respectivement); les prévisions faites à partir des équations de régression sont peu utilisables à cause de la largeur indue des intervalles de confiance.

Si on compare les TAAM de chacun des modes de transport, et pour la période 1940 à 1985 , seuls les taux d'accroissement de l'avion (avec 9.5\%) et de $\mathrm{l}^{\prime}$ autobus (avec $7.6 \%$ ) demeurent positifs. Le TAAM du rail est négatif (avec $-2.7 \%$ ) et aussi celui des véhicules automobiles (avec $-0.15 \%$ ). La principale leçon de la longue période est que la demande pour l'autobus et l'avion tend à remplacer le rail et l'automobile.

\section{Les tendances de courte durée (1976-1985)}

Les tendances de courte durée montrent peu de changement par rapport aux tendances longues. Les indices du tableau 2 (base 100 = 1976) donnent une bonne idée de l'évolution de la demande entre les années 1976 et 1985: le nombre de véhicules automo- 
biles diminue de façon sensible (perte de 18 points) entre ces deux dates. Pendant la même période, les voyageurs par rail augmentent quelque peu mais leur faible nombre $(38,012$ en 1985$)$, par rapport au total des visiteurs, laisse croire que ce mode de transport est en sursis.

Le transport par autobus s'est développé relativement bien de 1976 à 1985; trois années marquent cette période (1981-1982-1983). L'avion est le mode de transport qui a connu la meilleure performance; seule I'année 1983 est plus faible que les autres. Le graphique no 5 illustre bien la suprématie de l'avion et à un degré moindre de l'autobus face à l'automobile qui est en perte de vitesse pendant cette période.

Entre 1976 et 1985 , le TAAM pour les entrées de véhicules automobiles a êté do $-2.1 \%$; c'est une décroissance relative ment faible mais qui indique bien la baisse d'intérét pour ce mode de transport. Pour l'autobus, le TAAM est de $1 \%$ pendant cette période et de $3 \%$ pour l'avion. Ainsi, dans l'ensemble, il y a eu une certaine stabilisation de la demande de transport et celle-ci coincide avec l'évolution des arrivếes des touristes internationaux ces dernières années ${ }^{(17)}$,

Pendant les périodes 1976-1985, la part de marché du transport aérien est passée de $19.1 \%$ à $26.1 \%$ du total; dans la même période, le transport automobile a chuté de $63.8 \%$ a $54 \%$ du marché. Ainsi, les tendances récentes ne font que perpétuer les tendances de longue durée. On assiste à la quasi-disparition du rail comme mode de transport et aussi au plafonnement de l'automobile comme façon privilégiée de voyager pour les touristes américains. L'autobus et l'avion sont en constante progression malgré une certaine saturation de la demande touristique globale.

\section{L'horizon de I'an $\mathbf{2 0 0 0}$}

Dans le futur, les tendance de la demande de transport, décrites plus haut, pour les voyageurs américains visitant le Québec, subiront peu de modifications. On peut s'attendre (voir le tableau 3) à ce que, à moins de changements technologiques importants, le rail maintienne sa présence symbolique d'environ 40,000 voyageurs étrangers en moyenne, par année, et conserve une faible part du marché global du transport.

L'avenir de l'automobile comme moyen de transport pour les voyageurs américains n'est pas très rose: la plupart des prévisionnistes s'attendent à une saturation du parc automobile vers l'an 2000 et ce, pour l'ensemble des pays occidentaux ${ }^{\text {(18) }}$.

La crise énergétique des années 1973-1975 a entraîné une rupture dans les comportements des touristes par rapport à l'automobile utilisée jusqu'alors comme mode
TABLEAU 3

Évaluation de la demande de transport des voyageurs américains au Québec dans les prochaines années

\begin{tabular}{|c|c|c|c|}
\hline \multirow{2}{*}{ Modes } & \multicolumn{3}{|c|}{ Formes de développement } \\
\hline & Longue durée & Courte durée & Futur \\
\hline Rail & $\begin{array}{l}\text { Décroissance } \\
\text { forte }\end{array}$ & $\begin{array}{l}\text { Décroissance } \\
\text { faible }\end{array}$ & $\begin{array}{l}\text { Décroissance } \\
\text { faible }\end{array}$ \\
\hline Automobile & $\begin{array}{l}\text { Croissance puis } \\
\text { plafonnement }\end{array}$ & Plafonnement & $\begin{array}{l}\text { Décroissance } \\
\text { faible }\end{array}$ \\
\hline Autobus & $\begin{array}{l}\text { Croissance } \\
\text { moyenne }\end{array}$ & $\begin{array}{l}\text { Croissance } \\
\text { faible }\end{array}$ & $\begin{array}{l}\text { Croissance } \\
\text { faible }\end{array}$ \\
\hline Avion & $\begin{array}{l}\text { Croissance } \\
\text { forte }\end{array}$ & $\begin{array}{l}\text { Croissance } \\
\text { moyenne }\end{array}$ & $\begin{array}{l}\text { Croissance } \\
\text { forte }\end{array}$ \\
\hline
\end{tabular}

quasi-unique de transport. Les effets de cette crise apparaissent à plusieurs niveaux $x^{(19)}$ :

- les automobiles deviennent plus petites, moins confortables et possedent un rayon d'action plus réduit tant au plan physique que psychologique;

- réduction de la vitesse sur les grandes routes;

- le mixage des moyens de transport: avion/auto, avion/rail/auto, avion/ bateau de croisière/auto, etc.;

- valorisation d'une destination unique plutốt que le voyage itinérant.

On peut s'attendre (voir tableau 3) à une décroissance lente de l'automobile (en chiffres relatifs) comme moyen de transport touristique pour les voyageurs américains se rendant au Québec. La part de marché de ce mode de transport pourrait être inférieure à $50 \%$ dans les prochaines années.

L'avenir de l'autobus et l'avenir de l'avion comme modes de transport semble paradoxalement liés. L'autobus devrait connaître une croissance lente et stable et l'avion une croissance forte et prolongée jusqu"a l'an 2000.

Les études démontrent que I'autobus est sensible aux variations de temps alors que: "Peu sensible au temps, l'avion l'est au coût, suivi par l'automobile et l'auto$\operatorname{car}^{+4(20)}$. Ces deux modes de transport, l'avion et l'autobus, correspondent donc à des clientèles différentes. La clientèle qui utilise l'autobus, pour voyager, est beaucoup plus intéressée par le prix que par le "temps perdu" alors que la clientèle du transport aérien accorde une grande valeur au "temps gagné" par ce mode de transport plus rapide.

L'élasticité des prix de la demande pour le transport aćrien est assez élevée en ce qui concerne les voyages de loisir et de vacances $^{(21)}$. L'ensemble de ces facteurs expliquent pourquoi l'avion prendra une part de plus en plus grande du marché des transports.
Ainsi, dans les cinquante dernières années, on a vu, dans les modes de transport utilisés par les voyageurs Américains se rendant au Québec, la lente agonie du rail, la montée de l'automobile et son plafonnement. l'essor de l'autobus et de l'avion.

Chacun de ces modes de transport développait une forme particulière de tourisme. $\mathrm{L}^{+}$automobile favorisait une plus grande autonomie et des possibilités de découverte pratiquement sans limite. Le rail et l'avion et, à un moindre degré, l'autobus déterminent des destinations uniques ou, et c'est le moindre mal, l'usage des corridors tourégions.

Selon Jean Viard: "Chaque système de transport produit un dispositif touristique mode, les autres systèmes de transport tentent de $s^{\prime}$ adapter ${ }^{+}(22)$. L'automobile ne sera done plus que le prolongement de moyens de transport plus rapide, plus performant, le train à grande vitesse et l'avion. Il faut donc s'attendre à moyen et à long termes à de profonds bouleversements dans le monde des transports touristiques.

Une politique cohérente des transports touristiques devrait encourager le mixage maximum des divers modes de transport. Cette politique devrait inciter les intervenants de l'industrie touristique à inclure un mixage optimal des modes de transport dans tous les types de forfait. La mobilité condition vitale du développement de toutes les régions touristiques. $f$

\section{Références}

(1) ROUSSEAU, P. Histoire des transports, Favard, Paris, $196 \mathrm{t}$

(2) GLAZEBROOK, G.P., A history of transportation in Canada, volumes I et II, McClelland and Ste. wart Limited, Toronto, 1970.

(3) BRIERE, A. 'Les grands traits de /évolution du fourisme azu Québec" "Bullatin de l"Association des géographes de l'Amérique française, no 14 . septembre 1967 , pp. 83-95.

(Suite à la page 42 ) ristiques classiques au détriment des autres différent. Puis, quand celui-là est à la maximale pour les touristes devient une 
WHEATCROFT, Stephen, "The changing economics of international air transport ", Tourism Management, vol. 3, no 2, (June 1982), pp. 71-82.

\section{Transport maritime}

HANBY, Betsy, "1985 outlook for cruise lines'", in 1985 outlook for travel and tourism: proceedings of the US Travel Data Center - 1985 Travel outlook forum, Washington, D.C., USTDC, 1985, pp. 115-119.

CANADA, Bureau des conseillers en gestion, The tourism industry and the role of ferry services, Ottawa, BCG, 1976, $71 \mathrm{p}$.

KANE, Arthur W, and Jean-Claude POTIER, "The cruise catalysts", in Travel and Tourism Research Association Fifteenth Annual Conference, Salt-Lake City, TTRA, 1984, pp. 171-181.

LEWIS, Jay and Dan SAREL, "Cruise industry in the USA: matching supply and demand by 1990 " Travel and Tourism Analyst, (August 1986), pp. 43-55.

LUCAS GIRARDVILLE, Jean-Marie, Les croisières au Québec, Québec, Ministère du Tourisme, $1980,243 \mathrm{p}$.
MARTI, Bruce E.., "Cruising: smallvessel population characteristics" "Journal of Travel Research, vol. XXIV, no 4 (Spring 1986), pp. 25-28.

\section{Automobile et tourisme}

ASPECT ASSOCIATES, U.S. automobile travel to Canada 1972-1983: an econometric evaluation, Toronto, Aspect Associates for Tourism Industry Association of Canada, 1984,56 p.

BREDEMEIR, Judi, "The US car rental industry: surviving the 1980's'". Travel and Tourism Analyst (March 1986), pp. 47-55.

BRYAN, William, "Improved mileage discretionary income and iravel for pleasure", Journal of Travel Research, vol. XX, no 1 (Summer 1981), pp. 28-29.

CORSI, Thomas M. and Milton E. HAWEY, "Changes in Vacation Travel in Response to Motor fuel Shortages and higers prices", Journal of Travel Research, vol. XVII, no 4 (Spring 1979), pp. 7-11.

DIONNE, France, Rapport du comité Dionne sur les haltes routières, Québec, 1986.
KAMP, B. Dan, John L. CROMPTON and David M. HENSARLING, "The reactions of travelers to gasoline rationing and to increases in gasoline prices", Journal of Travel Research, vol. XVIII, no 1 (Summer 1979), pp. 37-41.

QUÉBEC (Province), Ministère des Transports et Ministère du tourisme, Projet de politique de signalisation du produit touristique, Québec, 1984, 62 p.

TRENT, Roger B. and Cecil POLLARD, "Gasoline prices and Discretionary auto travel: evidence from a panel survey", Journal of Travel Research, vol. XXI, no 4 (Spring 1984), pp. 12-16.

WILBUR, Allan, "Oulook for awto vacation market segments", in 1985-86 outlook for travel \& tourism: proceedings of the eleventh annual travel outlook forum, Washington, D.C., US Travel Data CEnter, 1986, pp. 112-124, f
La demande de transport

(Suite de la page 29)

(4) JAKLE, A. The tourist, travel in twentiethcentury North-America. University of Nebraska Press, Lincoln, 1985.

(5) WILSON, A., "A Statistical Theory of Spatial Distribution Models". Transportation Research. vol. I, N.Y, 1967 .

(6) OUANDT, R. The Demand for Travel: Theory and Measurement, Heath Lexington Books, Lexington, Mass., 1970.

(7) GOUDWIN, P., La valeur du temps, C.E.M.T. Paris, 1976.

(8) COOLEY, T,F, Forecasting the demand for travel, U.S. Department of Transportation, Washington, 1979.

(9) STOPHER, R.، MEYBURG, A., Behavioral Travel Demand Models, Lexington Books, Toronto, 1976.

(10)BROG, $W$, ROGERS, $K$, New Horizons in Travel-Behavioral Research, Lexington Books, Toronto, 1981

(11) BRAUDEL, F., "La longue dusée", in Eerits sur I'histoire, Flammarion, Paris, 1969 , p. 50.

I12IBAAUDEL, F., op. cit., p. 46 .

(13) NAHON, G. SALOMON, M., L'élaboration des prévisions de marché, Dunod, Paris, 1977, p. 21.

[14/Voyages entre le Canada et les autres pays, Statistique Canada, Cataloques (66-001).

(15) II s'agit ici du taux doaccroissement annuel moyen (TAAM) calculé avec la movenne géométrique.

(16)Les cing formes d'ajustement ont été calculées pour les quatre modes de transport, aै l'aide du progiciel S.A.S. sur l'ordinateur. Amdahl de l'Université du Québec à Montréal.
(17)BOER.JAN, P NANHOVE, N., "The Tourism Demand Reconsidered in the Context of the Eca nomic Crisis", in Revue de Tourisme, vol. 39, no 2. Berne, 1994

I18ISTUBBS, P., TYSON, W., DALVI, M., Transport Economics, George Allen Umwin, London, 1980, pp. $12-19$

I19ICHRISTIE, A.., MORRISON, A., The Tourism System, Prentice Hall, N.J., 1985, pp. 169-1970.

(20) LANG, R., Train, avrion ou automobile! Les choix de l'usager, Economica, Paris, 1990, p. 131

(21)BONSOR, N. Transportation Economics, Butterworths, Toronto, 1984 , pp. 56-58.

(22) VIARD, J., Penser les vacances, Actes-Sud, Marseille, 1984, p. 127
Pour une politique...

(Suite de la page 34)

\section{Dans les haltes routières}

Des bureaux d'informations touristiques sont déja localisés dans certaines haltes routières du ministère des Transports. Le ministère du Tourisme compte implanter, en collaboration avec les Associations touristiques régionales, des relais d'information touristique dans des haltes routières situés à des endroits stratégiques. Les relais d'information touristique sont en fait des panneaux informatifs et promotionnels. Ils visent par la transmission d'informations à assurer un sentiment de suivi entre le touriste et le réseau de bureaux d'informations touristiques. Ils s'intègrent également à la signalisation touristique, car ils ont aussi comme objectif d'aider à acheminer la clientèle touristique jusqu'à l'attrait, l'activité ou le service choisi.

D'ores et déjà, plus de gens trouveront satisfaction face à la signalisation touristique. Nous croyons que les démarches entreprises contribueront à rendre le Québec touristique plus accueillant. $f$

(1) LAVOIE Danielle, Vers une nouvelle signalisation routière du produit touristique québécois. Ministère de I'lndustrie, du Commerce et du Tourisme, 1983, 32 pagess.

(2) Ministère des Transports, Ministëre du Tourisme La signalisation du produit touriatique quobbcois. 1987, 15 pages. 